\title{
Tactile sensitivity of the mouse fetus
}

\author{
H. RICHARD SCHIFFMAN and CAROLYN A. MCHALE \\ Rutgers-The State University, New Brunswick, New Jersey
}

\begin{abstract}
We used von Frey hairs to assess the tactile sensitivity of various bodily loci in 13-17-day-old mouse fetuses. Regardless of age, more fetuses displayed evoked activity in response to stimulation applied to the nose than to any other locus. The proportion of fetuses responding to stimuli applied elsewhere increased significantly with age. Stimulation of the nose also yielded the lowest threshold for fetuses of all ages. The next lowest threshold was obtained from stimulation of the paws, followed by stimulation of the head and neck. No pattern of change in thresholds of the various regions was observed from Day 15 to Day 17 . In a second experiment, we compared the tactile sensitivity of control fetuses to that of fetuses having resided singly in the uterus. Although singleton status enhances several aspects of development, it had no effect upon the development of tactile sensitivity.
\end{abstract}

Fetuses of many mammalian species, including humans, display motility in response to tactile stimulation (Angulo y Gonzalez, 1932; Barcroft \& Barron, 1939; Bodian, Melby, \& Taylor, 1968; Hooker, 1936; Narayanan, Fox, \& Hamburger, 1971; Windle \& Griffin, 1931). Such evoked behavior has been used to study neurological development-specifically, the emergence of sensorimotor function. While providing important information pertaining to the responsivity of fetuses of varying gestational ages to stimulation, the earlier research did not systematically assess changes in tactile threshold during development. In previous studies, fetuses were challenged with but a few stimuli, which typically provided intense, clearly suprathreshold, pressure stimulation. Carmichael (1934), in his classic monograph, used von Frey hairs to stimulate fetal guinea pigs, but the intensities of the stimuli were not specified and they were not applied systematically.

In the present investigation, we sought to determine the tactile threshold of fetuses as a function of gestational age and locus of stimulation. We also attempted to determine whether the development of tactile sensitivity is affected by singular occupancy of the uterus. Such singular prenatal status increases several aspects of biobehavioral maturation. For example, Van Marthens, Grauel, and Zamenhof (1972) removed all but a single rabbit conceptus soon after coitus. Just prior to term, the singleton fetuses were up to $105 \%$ heavier than controls. Moreover, their cerebral DNA content was elevated by as much as $21 \%$. Singleton rat fetuses have elevated body, placental, and brain weights (Croskerry, Smith, Hall, \& Shepard, 1978; Van Marthens \& Grauel, 1974). More recently, Gandel-

This research was supported by a grant from the Research Council of Rutgers-The State University. Thanks are extended to Ronald Gandelman for his suggestions, support, and guidance at all phases of this investigation, and to Suzanne Griest-Bousquet for her comments on the manuscript. Please address all correspondence to H. R. Schiffman, Department of Psychology, Rutgers-The State University, Busch Campus, New Brunswick, NJ 08903. man and Graham (1986) found that singleton mouse fetuses are heavier at birth and exhibit enhanced durations and frequencies of spontaneous fetal activity than do controls. Mice that have been singletons also walk, maintain their grip, and display eye and vaginal opening earlier than do controls. It has been suggested that the singletons' advanced rate of development is related to placental morphology. Placentas of singletons are larger than those of controls (McLaren, 1965; Zambrana \& Greenwald, 1971), leading to enhanced nutrient and oxygen transfer and more efficient removal of waste products. It remains to be determined if the development of tactile sensitivity is similarly enhanced by singleton status or whether it emerges in a relatively invariant manner, impervious to potential developmentally augmenting influences.

\section{GENERAL METHOD}

\section{Animals}

Rockland-Swiss albino mice, maintained as an outbred strain in a closed colony, were kept on a 12:12 h light:dark cycle with lights on at $0600 \mathrm{~h}$. They were provided with unlimited access to food and water.

\section{Procedure}

Sixty- to 70-day-old virgin females were group-housed with males and examined daily between 0800 and $0900 \mathrm{~h}$ for copulatory plugs, the discovery of which signified Day 0 of pregnancy. With this procedure, the gestation period is 19 days. Pregnant animals were housed singly in $12.7 \times 17.8 \times 27.9 \mathrm{~cm}$ translucent cages with wood shavings on the floors.

Spinal transection was used to prepare the pregnant animal for fetal observation (see Smotherman, Richards, \& Robinson, 1984). On the appropriate day of gestation, the pregnant mice were anesthetized with ether and their spinal cords were transected at Level T4. Etherization was then discontinued. This procedure causes paralysis of the lower extremity and eliminates tactile sensitivity of that region. The spinalized mice were secured to a contoured plate and immersed from the neck down in Locke's solution maintained at $37^{\circ} \mathrm{C}$. An incision was made in the abdominal wall to expose the uterus of the pregnant females. A fetus or fetuses, chosen at random, was/were removed from the uterus with extreme care taken to ensure that both the amniotic membrane and the attachment of the umbilical cord to the placenta remained intact. (This methodology is similar to that of Kodama \& Sekiguchi, 1984, and 


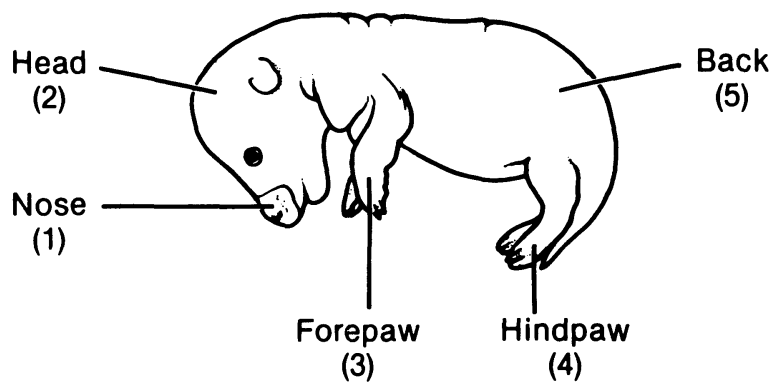

Figure 1. Schematic presentation of the mouse fetus showing sites of stimulation and the order in which they were stimulated (adapted from Narayanan et al., 1971).

Narayanan et al., 1971.) The application of tactile stimuli, made through the transparent amniotic membrane, commenced $10 \mathrm{~min}$ after the fetuses were exposed. Since a minimum of $5 \mathrm{~min}$ was required to prepare the animal, at least 15 min elapsed between removal of the ether and data collection. Kirby (1979) reported that rat fetuses require 15-20 min to recover following etherization of the dam.

Previous work (Gandelman, unpublished observations) has revealed that if an exteriorized mouse fetus retains its normal pink coloration, rather than becoming blue-gray in appearance, oxygenation is adequate. Therefore, only fetuses displaying pink coloration prior to and throughout the testing period were used ( $77 \%$ of the total number of fetuses).

A pressure aesthesiometer (Stoelting Co., Chicago) with a range of $0.45-1,480 \mathrm{mg}$ of force was used to provide tactile stimulation of varying intensities to the body locations diagrammed in Figure 1. (Stimuli were applied in the order indicated in the figure.) The device consists of a series of Teflon filaments of various diameters. The tip of the filament was pressed against a site until the filament bowed, and was left in place for $5 \mathrm{sec}$, during which the fetus was observed for an evoked response to the tactile stimulus. No attempt was made to differentiate between mass movement (i.e., movement of the entire trunk) and local movement (i.e., movement of only the body part stimulated). Stimulation was applied only during periods when neither the fetus nor the mother was moving.

The stimuli were applied in an ascending series beginning with a filament that, based upon the results of a pilot study, is known to be subthreshold for all stimulation sites. After each site was stimulated, the filament with the next highest diameter was used. The interval between stimulus presentations was approximately $25 \mathrm{sec}$, unless the fetus and/or the dam was moving. Thus, the next stimulus was not applied until spontaneous motility ceased. This procedure continued until stimulation of a given region evoked a response or until the fetus proved completely unresponsive to stimulation of that site. After all stimulation sites were assessed, the procedure was repeated. Surprisingly little variation occurred between the two series. In the few cases (less than 5\%) in which a response was elicited on one series but not on the other, a third series was given. If a response was evoked on the third series, the site was considered positive. A third test was also given if a stimulation site yielded different thresholds on the previous series. In that case, the threshold was determined by the majority results offered by the third series. (In no case were three different thresholds obtained.) At the conclusion of testing, the 15-17-day-old fetuses were sexed. (The sex of 13- and 14-day-olds cannot be determined accurately without performing histology.)

\section{EXPERIMENT 1 EVOKED BEHAVIOR AND FETAL AGE}

\section{Method}

Eight pregnant primiparous animals were assigned to each of five groups differentiated as to day of gestation. They were Days 13-17. (Because of the relatively large size of 18-day-olds, it is extremely difficult to successfully remove them from the uterus while leaving the amniotic membrane intact. Therefore, they were not used here.) On the appropriate day, the animals were prepared as described above. Two fetuses, one from each uterine horn, were selected at random. Each of the five groups, therefore, was composed of 16 fetuses.

\section{Results}

Evoked activity elicited by stimulation of at least one region was displayed by all 16 of the 14-17-day-old fetuses but by only half of the 13-day-olds. The percentage of fetuses that responded as a function of stimulation site (irrespective of stimulus intensity) is shown in Figure 2 (only data from the 8 13-day-olds that displayed evoked activity are included). Summing over age, the vast majority of fetuses $(96 \%)$ displayed greater activity in response to stimulation applied to the nose than to any other location $\left[\chi^{2}(4)=87.4, p<.001\right]$. In addition, with the exception of stimulation of the nose, the proportion of fetuses that responded to stimuli applied to all other locations increased with advancing age $\left[\chi^{2}(4) \geq 14.05\right.$, $p s<.01]$. Finally, neither gender differences nor similarities between pairs of fetuses from the same uterus were observed. With regard to the latter factor, the probability of 1 fetus responding to a particular stimulus was independent of the behavior of the other.

Threshold data are shown in Figure 3. Because the proportion of 13-16-day-old fetuses that responded to

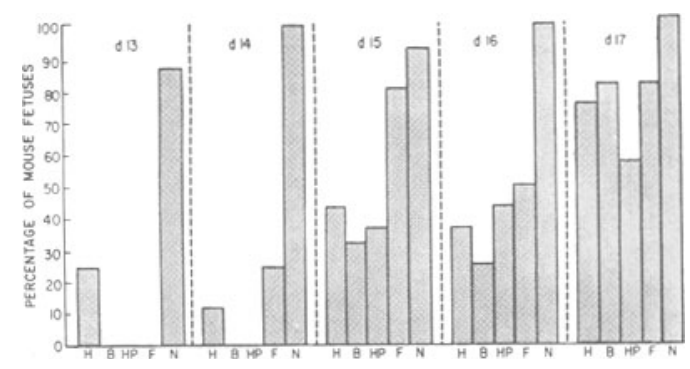

Figure 2. Percentage of 13-17-day-old mouse fetuses that displayed activity in response to stimulation of the head $(\mathrm{H})$, back $(\mathrm{B})$, hindpaw (HP), forepaw (F), and nose (N). Each age group contained 16 fetuses.

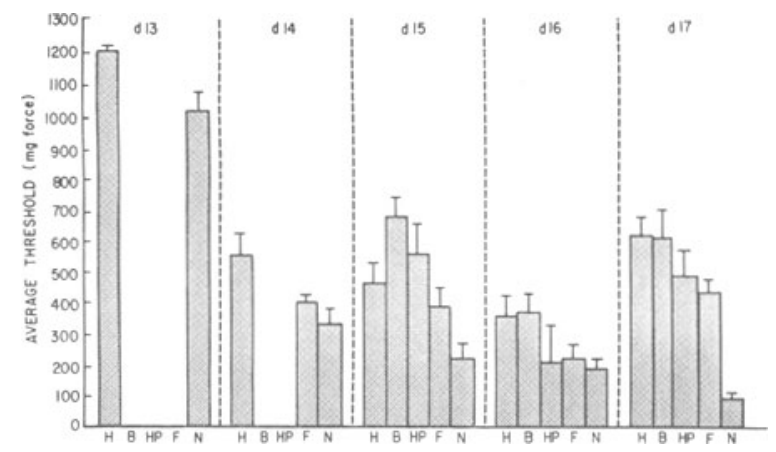

Figure 3. Average threshold (and SEM) of 13-17-day-old mouse fetuses to respond to tactile stimulation as a function of stimulation site. $\mathbf{H}=$ head, $B=$ back, $H P=$ hindpaw, $F=$ forepaw, and $N$ $=$ nose. Each age group contained 16 fetuses. 
stimulation of the head, back, and hindpaw was relatively low (see Figure 2), an overall statistical analysis of threshold data with age and stimulation site as factors was not possible. Nevertheless, two conclusions may reasonably be drawn from visual inspection of the data. First, no obvious pattern of change in thresholds of the various body regions was observed from Day 15 to Day 17 . Second, the nose was the region most sensitive to pressure stimulation at all ages.

\section{EXPERIMENT 2 EVOKED BEHAVIOR OF THE SINGLETON FETUS}

\section{Method}

The fetuses were removed from a group of timed-mated 8-day-pregnant nulliparous mice as described below; another group was subjected to a control procedure consisting of all aspects of the surgical procedure except for the actual removal of embryos. The former animals were anesthetized with ether, a midline incision was made in the abdominal wall, and both uterine horns were exposed. Incisions of approximately $1.5 \mathrm{~mm}$ were made in the uterus above all but one randomly selected embryo, which was designated to be the singleton. All but this embryo, including placental tissue, were removed with forceps and the uterine horns were returned to the body cavity.

Each of the two groups formed two subgroups of 10 animals; one was prepared for fetal stimulation and observation on Day 14 of gestation and the other on Day 16. The testing procedure was the same as that of Experiment 1, with one exception applicable to the control animals: immediately following immersion in Locke's solution and exteriorization of the uterus, all but a single randomly chosen fetus was removed. This was accomplished by slowly rotating the fetuses and severing them from the maternal unit, a procedure that almost always produced negligible bleeding. In the few cases in which significant bleeding followed, the preparation was terminated. Removal of all but a single fetus controlled for possible nondevelopmental, concurrent effects of singleton status, such as alteration of blood flow.

\section{Results}

Table 1 presents the proportion of 14- and 16-day-old singleton and control fetuses that exhibited activity in response to the application of tactile stimulation. There were no statistically significant differences for either age group at any stimulation site.

Threshold data are shown in Figure 4. Regardless of fetal age, singleton status did not influence the threshold for the evocation of activity in response to tactile stimulation.

Table 1

Number of 14- and 16-Day-Old Singleton and Control Fetuses Responding to Tactile Stimulation of the Head, Back, Hindpaw, Forepaw, and Nose

\begin{tabular}{lccccc}
\hline & \multicolumn{5}{c}{ Stimulation Site } \\
\cline { 2 - 6 } Subjects & Head & Back & Hindpaw & Forepaw & Nose \\
\hline \multirow{5}{*}{ Singletons } & 9 & 7 & 9 & 10 & 10 \\
Controls & 5 & 3 & 6 & 7 & 10 \\
& \multicolumn{7}{c}{16 -Day-Olds } \\
Singletons & 9 & 10 & 10 & 10 & 10 \\
Controls & 9 & 9 & 10 & 10 & 10 \\
\hline
\end{tabular}

Note $-n=10$ in each group.

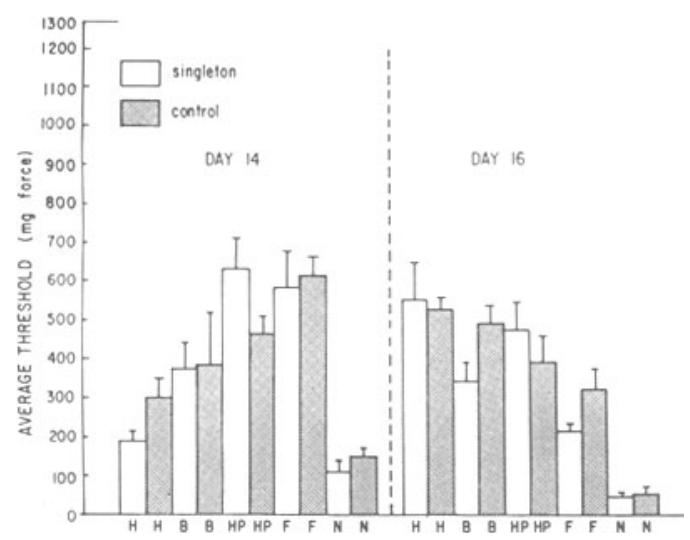

Figure 4. Average threshold (and SEM) of 14- and 16-day-old singleton and control fetuses to respond to tactile stimulation as a function of stimulation site. $\mathbf{H}=$ head, $B=$ back, $\mathbf{H P}=$ hindpaw, $F=$ forepaw, and $N=$ nose. Each of the four groups contained 10 fetuses.

\section{GENERAL DISCUSSION}

Several points of a cautionary nature must be considered. One concerns the fact that the tactile stimuli were not applied directly to fetal tissue. Rather, they were applied to the overlying amniotic membrane, thus raising the possibility that the membrane served to attenuate responsiveness of the fetus to the stimuli.

A related point concerns the fact that the volume of amniotic fluid decreases with advancing gestational age (Bradley \& Mistretta, 1975). Therefore, the younger the fetus, the more of a buffer between it and the stimulus, thereby possibly causing a spurious elevation of response threshold. Although this problem appears to be rectifiable simply by removing the fetus from its amniotic sac, such a procedure could not be employed here because "shelled" fetuses (with uninterrupted umbilical circulation) exhibit a gasping response and asphyxia, and expire quickly (Gandelman, unpublished observation).

A second point concerns spontaneous fetal activity. Stimuli were applied during periods of fetal quiescence, and a response had to occur within the ensuing $5 \mathrm{sec}$ for it to be considered an evoked activitythat is, a response to that stimulus. Even though each fetus was tested twice (and some three times), it is impossible to state categorically that all of the behaviors classified as being evoked did not occur spontaneously.

A final point concerns the order of testing. Identical testing orders were used in an effort to treat the fetus groups identically. With testing order held constant, one can compare with greater confidence the responsiveness of the 13-17-day-old animals in Experiment 1 and the singleton and nonsingleton fetus groups in Experiment 2.

The data show that the mouse fetus, like that of other species, exhibits activity in response to the application of tactile stimulation. The proportion of fetuses displaying evoked activity generally increased between Days 13 and 17 of gestation (see Figure 2). It is unlikely that this increase is mediated by maturation of the motor system per se, because by Gestation Day 14 mouse fetuses spontaneously exhibit all of the behaviors seen in response to tactile stimulation (Gandelman \& Graham, unpublished data). Therefore, it appears that sufficient maturation of relevant receptors, afferent neural mechanisms, the cortical receiving area, or a combination of such factors occurs between Days 13 and 17.

The application of tactile stimulation to the nose, in addition to evoking activity from practically all fetuses, also yielded the lowest thresholds, irrespective of fetal age (see Figure 3). By Gestation Day 16, the next lowest thresholds were produced by stimulation of the paws, followed by stimulation of the head and back. This pattern of prenatal tactile development coincides with the basic survival needs of the organism at 
birth. Thus, "cutaneous sensitivity around the mouth and snout is well developed, in the absence of which the organism would be unable to locate the teat and suck" (Narayanan et al., 1971, p. 128). It is interesting that this trend, observed across body loci for the fetal mouse, coincides with that obtained from the adult human (Weinstein, 1968). Indeed, one would expect for most terrestrial vertebrates that the guiding and exploratory parts of the body-that is, the more mobile and functionally relevant regions-would be most sensitive to mechanical encounters with the environment, and would accordingly manifest lower tactile thresholds.

A comparison of the results of Experiments 1 and 2 reveals differences that may be attributable to nondevelopmental aspects of residing singly in the uterus. Inspection of Figure 2 and Table 1 reveals that a greater proportion of 14-day-old singleton and control fetuses responded to stimulation of the head than did fetuses of the first experiment. A similar difference also applies to stimulation of the back and hindpaws of 16-day-olds. In addition, the thresholds of controls in Experiment 2 were generally greater than those of the controls used in Experiment 1. Since the control fetuses of Experiment 2 were also singletons, but prepared just prior to testing, the elevation in the proportion of responding fetuses in Experiment 2 may be due to a nondevelopmental component of singleton status, such as enhanced blood flow.

That singleton status did not influence response thresholds to tactile stimulation was surprising, because sole occupancy of the uterus in polytocous species markedly affects the facets of development described above. This finding prompts one to ask whether the maturation of tactile sensitivity is impervious to other or all maneuvers that enhance development. Moreover, one must consider if maturational capacity is also unresponsive to manipulations that retard development. That is, it is of interest to consider that the development of tactile sensitivity is a uniquely independently driven process, generally unaffected by both endogenous and exogenous perturbations.

\section{REFERENCES}

Angulo y Gonzalez, A. W. (1932). The prenatal development of behavior in the albino rat. Journal of Comparative Neurology, 55, 395-442.

BARCROFT, J., \& BARRon, D. H. (1939). The development of behavior in fetal sheep. Journal of Comparative Neurology, 70, 477-502.

Bodian, D., Melby, E. C., JR., \& TAYlor, N. (1968). Development of fine structure of spinal cord in monkey fetuses: II. Prereflex period to period of long intersegmental reflexes. Journal of Comparative Neurology, 133, 113-166.

Bradley, R. M., \& Mistretta, C. M. (1975). Fetal sensory receptors. Physiological Review, 55, 352-382.
Carmichael, L. (1934). An experimental study in the prenatal guinea pig of the origin and development of reflexes and patterns of behavior in relation to the stimulation of specific receptor areas during the period of fetal life. Genetic Psychological Monographs, 16, 337-491.

Croskerry, P. G., Smith, G. K., Hall, S., \& ShePard, B. J. (1978). Fetal and placental growth in the rat following differential reduction of litter size. Biology of the Neonate, 33, 31-38.

Gandelman, R., \& Graham, S. (1986). Development of the surgicallyproduced singleton mouse fetus. Developmental Psychobiology, 19, 343-350.

HoOKER, D. (1936). Early fetal activities in mammals. Yale Journal of Biology \& Medicine, 8, 579-602.

KIRBY, M. L. (1979). A quantitative method for determining the effect of opiates on fetal rats in utero. Problems of Drug Dependence [NIDA Research Monographs], 27, 191-197.

Kodama, N., \& SekIGUCHI, S. (1984). The development of spontaneous body movements in prenatal and perinatal mice. Developmental Psychobiology, 17, 139-150.

MCLAREN, A. (1965). Genetic and environmental effects on fetal and placental growth in mice. Journal of Reproduction \& Fertility, 9, 79-98.

Narayanan, C. H., Fox, M. W., \& Hamburger, V. (1971). Prenatal development of spontaneous and evoked activity in the rat (Rattus Norwegicus albinus). Behaviour, 40, 100-134.

Smotherman, W. P., Richards, L. S., \& Robinson, S. R. (1984). Techniques for observing fetal behavior in utero: A comparison of chemomyelotomy and spinal transection. Developmental Psychobiology, 17, 661-674.

Van Marthens, E., \& Grauel, L. (1974). Ovarian, adrenal and litter restriction effects on fetal and placental development. Nutrition \& Metabolism, 17, 198-204.

Van Marthens, E., Grauel, L., \& Zamenhof, S. (1972). Enhancement of prenatal development by operative restriction of litter size in the rabbit. Life Science, 11, 1031-1035.

WeINSTEIN, $S$. (1968). Intensive and extensive aspects of tactile sensitivity as a function of body part, sex, and laterality. In D. R. Kenshalo (Ed.), The skin senses (pp. 195-222). Springfield, IL: Thomas.

WINDLE, W. F., \& GRIFFIN, A. M. (1931). Observations on embryonic and fetal movements of the cat. Journal of Comparative Neurology, 52, 149-188.

Zambrana, M. A., \& Greenwald, G. S. (1971). Effects of fetal, ovarian, and placental weight of various number of fetuses in the rat. Biology of the Neonate, 4, 216-223.

(Manuscript received January 19, 1990.) 